\title{
表面活性剂增强叶酸的光稳定性
}

刘鸣华

国家纳米科学中心, 北京 100190

\section{Surfactant Enhancing Photo-Stability of Folic Acid}

\section{LIU Minghua}

National Center for Nanoscience and Technology, Beijing 100190, P. R. China.

Email: liuminghua@nanoctr.cn; liumh@iccas.ac.cn.

Published online: November 30, 2018.

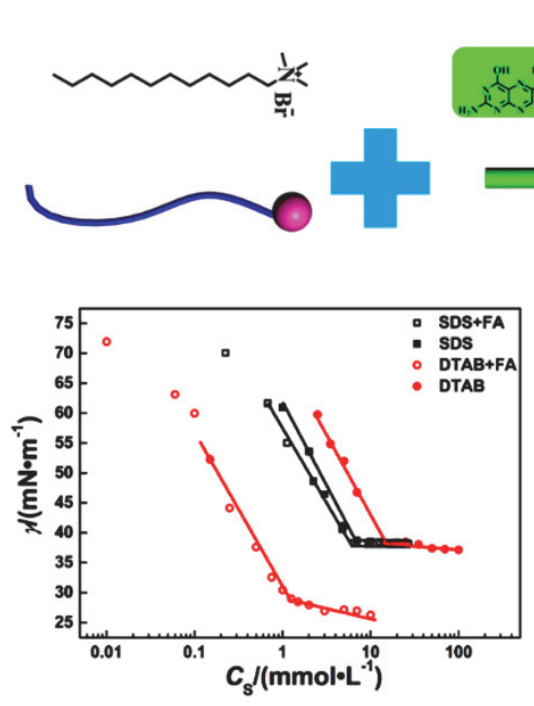

叶酸是一种参与人体多项生理活动, 对人体 有重要营养价值的水溶性B族维生素, 也是一种常 用的构筑功能材料的基本单元 ${ }^{1}$ 。然而, 叶酸的光 不稳定性限制了其实际应用 2 。许多研究证实只要 能为叶酸提供疏水空间而将叶酸包裹在其中，使 叶酸免于暴露在空气中, 就能在一定程度上提高 叶酸的光稳定性 3,4 。

表面活性剂是一种能够很好提供疏水空间的 载体, 已广泛应用于医药制剂中, 用以提高有机小 分子药物的溶解度、稳定性和生物相容性等 5,6 。此 外, 有机小分子的加入能够屏蔽表面活性剂极性 头基之间的静电排斥, 增强表面活性剂烷基链之 间的疏水相互作用，使得表面活性剂具有更低的 临界胶束浓度 $(\mathrm{CMC})$ 和更高的表面活性, 进一步

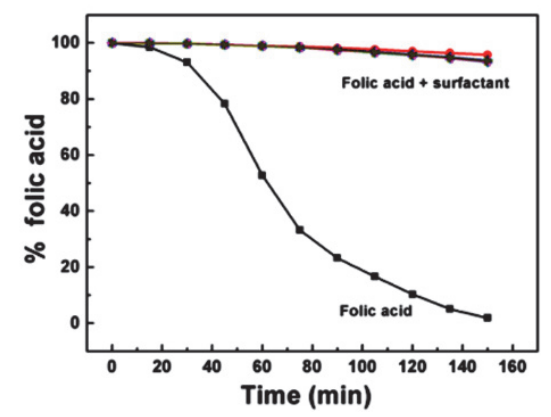

提高表面活性剂增溶与包裹小分子的能力 7,8 。表 面活性剂对有机小分子的增溶与包裹作用与表面 活性剂的分子结构有关, 因此, 为叶酸选择高效的 表面活性剂保护载体是一项有意义的工作。

中国科学院化学研究所王毅琳研究员等人详 细研究了叶酸与四种带有不同电荷、不同寡聚度 的表面活性剂的相互作用, 表面活性剂包括十二 烷基硫酸钠(SDS)、十二烷基三甲基溴化铵(DTAB), 连接基团为 6 个亚甲基的季铵盐型 Gemini 表面活 性剂(12-6-12) 以及线性季铵盐三聚表面活性剂 (12-3-12-3-12)。他们发现叶酸能明显提高阳离子型 表面活性剂的表面活性, 却对阴离子型表面活性剂 SDS 的表面活性几乎没有影响; 阴离子表面活性剂 SDS 抑制叶酸光氧化降解的效率较低, 而阳离子 
表面活性剂都能够显著抑制叶酸的光氧化降解, 且随着表面活性剂寡聚度的增加, 抑制效果增强, 所需表面活性剂的浓度显著降低, 寡聚表面活性 剂 12-3-12-3-12 的抑制效率高达 96\%。他们对不 同表面活性剂与叶酸的相互作用机理进行了详细 地研究, 认为表面活性剂分子的堆积方式, 即形成 聚集体的紧密程度是影响稳定效率的关键因素。

该工作已在物理化学学报上在线发表 (doi: 10.3866/PKU.WHXB201809038) ${ }^{9}$ 。该工作阐 明了不同结构的表面活性剂分子与叶酸相互作用 的差异以及提高叶酸光稳定性的差异, 为叶酸选 择了合适的保护载体。该工作对于调控表面活性 剂聚集行为、为不稳定小分子选择合适的载体具 有重要的启发意义。

\section{References}

(1) Off, M. K.; Steindal, A. E.; Porojnicu, A. C.; Juzeniene, A.; Vorobey, A.; Johnsson, A.; Moan, J. J. Photochem. Photobiol. B 2005, 80, 47. doi: 10.1016/j.jphotobiol.2005.03.001

(2) Akhtar, M. J.; Khan, M. A.; Ahmad, I. J. Pharm. Biomed. Anal. 1999,
19, 269. doi: 10.1016/S0731-7085(98)00038-7

(3) Vorobey, P.; Steindal, A. E.; Off, M. K.; Vorobey, A.; Moan, J. Photochem. Photobiol. 2006, 82, 817. doi: 10.1562/2005-11-23-RA-739

(4) Liang, L.; Subirade, M. J. Phys. Chem. B 2010, 114, 6707. doi: $10.1021 / \mathrm{jp} 101096 \mathrm{r}$

(5) Bhat, P. A.; Rather, G. M.; Dar, A. A. J. Phys. Chem. B 2009, 113, 997. doi: $10.1021 / \mathrm{jp} 807229 \mathrm{c}$

(6) Xie, H. J.; Liu, C. C.; Sun, Q.; Gu, Q.; Lei, Q. F.; Fang, W. J. Acto Phys. -Chim. Sin. 2016, 32 (12), 2951. [谢湖均, 刘程程, 孙强, 顾青, 雷群芳, 方文军. 物理化学学报, 2016, 32 (12), 2951.] doi: 10.3866/PKU.WHXB201609231

(7) Wang, M. N.; Wu, C. X.; Tang, Y. Q.; Fan, Y. X.; Han, Y. C.; Wang, Y. L. Soft Matter 2014, 10, 3432. doi: 10.1039/c4sm00086b

(8) Fan, Y. X.; Han, Y. C.; Wang, Y. L. Acta Phys. -Chim. Sin. 2016, 32 (1), 214. [范雅珣, 韩玉淳, 王毅琳. 物理化学学报, 2016, 32 (1), 214.] doi: 10.3866/PKU.WHXB201511022

(9) Luo, S. Q.; Wang, M. N.; Zhao, W. W.; Wang, Y. L. Acta Phys. -Chim. Sin. 2019, 35 (7), 766. [罗思琪, 王美娜, 赵微微, 王毅琳. 物理化 学学报, 2019, 35 (7), 766.] doi: 10.3866/PKU.WHXB201809038 